\title{
AVALIAÇÃO DO USO DE MODELOS ADITIVO E DE INTERAÇÕES NA PREVISÃO DA REATIVIDADE E DA RESISTÊNCIA MECÂNIICA DO COQUE*
}

\author{
Matheus Teixeira Fraga ${ }^{1}$ \\ Bruno Deves Flores ${ }^{2}$ \\ Eduardo Osório ${ }^{3}$ \\ Antônio Cezar Faria Vilela ${ }^{4}$
}

\section{Resumo}

É comum o uso de modelos matemáticos no intuito de prever a qualidade do coque, sendo a maioria dos modelos atualmente disponíveis baseada nas propriedades dos carvões. Para este trabalho foram feitas misturas binárias e ternárias de carvões com variados teores de matéria volátil, a fim de se produzir coque em escala laboratorial. $A$ reatividade ao $\mathrm{CO}_{2}$ em termobalança e resistência mecânica em tambores foi avaliada. Dois modelos para previsão de indicadores de qualidade do coque foram avaliados, o modelo aditivo e o modelo de interação. A partir da metodologia desenvolvida em laboratório foi possível produzir, avaliar e comparar coques feitos a partir de diferentes carvões e misturas. O modelo aditivo mostrou uma boa capacidade de prever a reatividade dos coques. No entanto, para os índices derivados dos testes de tambores os valores calculados mostram uma diferença importante em relação aos experimentais, indicando interação dos carvões no processo de carbonização. Nos resultados do modelo de interações, os valores calculados para reatividade e resistência mecânica, se comparados com os resultados do modelo aditivo, mostraram uma melhora na capacidade de previsão da qualidade do coque. O modelo de interações se apresenta como uma ferramenta interessante na seleção e mistura de carvões.

Palavras-chave: Coque; Reatividade; Resistência mecânica; Coke quality prediction.

\section{EVALUATION OF ADDITIVE AND INTERACTIONS MODELS TO PREDICT COKE REACTIVITY AND MECHANICAL STRENGTH}

\section{Abstract}

It is common to use mathematical models in order to predict the quality of coke, being most of the currently available models designed based on the properties of coals. For this work binary and ternary coal blends composed of coals with varied volatile matter yield were selected in order to produce coke in laboratorial scale. Coke reactivity towards $\mathrm{CO}_{2}$ and tumbler test were carried out. Two models to predict the coke quality parameters were assessed, the first is the additive model and the second the interaction model. From the methodology developed in the laboratory it was possible to produce, evaluate and compare cokes made from different coals and blends. The additive model showed a good capability to predict coke reactivity. However, the parameters taken from the tumbler tests the calculated values showed important differences in relation to the experimental values, indicating interaction in the carbonization process. Taking in to consideration the interaction model, the values calculated for reactivity and mechanical strength when compared to the results of additive model, the first model gives better prediction results. The interaction model is an interesting tool in the selection and blending of coals.

Keywords: Coke; Reactivity; Mechanical strength; Coke quality prediction.

\footnotetext{
1 Graduando, Laboratório de Siderurgia (LASID), Universidade Federal do Rio Grande do Sul (UFRGS), Porto Alegre, Rio Grande do Sul, Brasil.

2 Doutorando do PPGE3M, LASID, UFRGS, Porto Alegre, Rio Grande do Sul, Brasil.

3 Dr., Professor, LASID, UFRGS, Porto Alegre, Rio Grande do Sul, Brasil.

4 Dr-Ing., Professor, Coordenador do LASID, UFRGS, Porto Alegre, Rio Grande do Sul, Brasil.
} 


\section{INTRODUÇÃO}

Dentro dos altos-fornos, o coque deve desempenhar funções importantes, como gerar gases para a redução da carga ferrosa, fornece calor para o processo, além de dar suporte a toda a carga do reator carga, criando um leito poroso para a passagem de gases e líquido. Logo, a obtenção de um coque com qualidade é de fundamental importância para o bom funcionamento dos altos-fornos.

A qualidade do coque em ambientes industriais geralmente é avaliada através de uma série de testes, os quais em essência visam medir sua resistência à degradação, seja ela provocada por efeitos mecânicos, químicos, ou ainda uma combinação de ambos. Tradicionalmente a avaliação da resistência do coque a efeitos como abrasão e impacto é realiza da por meio de testes padronizados em tambores rotativos. No entanto, testes como esses não demonstram como o coque se comporta quando submetido a condições mais próximas dos altos-fornos. A Nippon Steel Corporation demonstrou através de estudos pioneiros [1] a necessidade de se introduzir novos parâmetros de qualidade, baseados na reatividade do coque ao $\mathrm{CO}_{2}$ (CRI- "CokeReactivity Index") e em sua resistência após a reação (CSR- "CokeStrengthafterReaction").

Visto que existe uma numerosa quantidade de variáveis a serem controlas no momento de se incorporar um determinado carvão na fabricação do coque, é pratica comum dentro das usinas siderúrgicas a utilização de modelos matemáticos para a previsão da qualidade do coque. A maioria dos modelos atualmente disponíveis baseada nas propriedades dos carvões. No entanto, devido à grande diversidade e heterogeneidade dos carvões utilizados para a fabricação de coque no mundo, não há um modelo com aplicabilidade universal, de modo cada usina deve buscar compor o modelo que melhor se ajuste aos carvões que utiliza [2].

A existência de uma numerosa quantidade de variáveis a serem controlas no momento de se incorporar um determinado carvão na fabricação do coque, é pratica comum dentro das usinas siderúrgicas a utilização de modelos matemáticos para a previsão da qualidade do coque. A maioria dos modelos atualmente disponíveis baseada nas propriedades dos carvões. No entanto, devido à grande diversidade e heterogeneidade dos carvões utilizados para a fabricação de coque no mundo, não há um modelo com aplicabilidade universal, de modo cada usina deve buscar compor o modelo que melhor se ajuste aos carvões que utiliza [2].

Uma das possibilidades mais simples para a previsão de indicadores de qualidade de coque é considerar que esses podem ser estimados por aditividade. Na literatura não há um consenso claro quanto a alguns desses fatores (DI, CSR e CRI) serem ou não aditivos [3;4;5]. Chiu (1982) relata a falta de aditividade para índices de resistência mecânica em tambor, mas classifica a reatividade como fator aditivo. Já Álvarez et al. (2007) acreditam que além da reatividade (CRI) e da resistência mecânica após a reação (CSR), os índices de resistência mecânica a frio do coque também podem ser estimados por modelos aditivos, mas com dispersões muito maiores.

O objetivo do presente trabalho foi avaliar a possibilidade do uso de um modelo aditivo para a previsão de indicadores de resistência mecânica e reatividade de coques produzidos em escala laboratorial. Além disso, testou-se no mesmo sentido o modelo interação, desenvolvido originalmente para identificar interações entre carvões [6]. 


\section{MATERIAIS E MÉTODOS}

\subsection{Carvões e Misturas}

Para este trabalho utilizou-se 3 tipos de carvões de origem americana, sendo eles divididos em $A$ - alto volátil, $B$ - médio volátil e $C$ - baixo volátil. Como se pode ver, na Tabela 1, as composições dos carvões de estudo apresentam reflectância entre 0,81 e 1,40 e fluidez variando de 56 a 15392 ddpm.

Tabela 1 - Composição dos carvões utilizados para o estudo.

\begin{tabular}{|c|c|c|c|c|}
\hline \multicolumn{2}{|l|}{ Análises } & A & B & C \\
\hline \multirow{4}{*}{$\begin{array}{c}\text { Imediata } \\
\text { (bs \% em massa) }\end{array}$} & MV & 34,8 & 21,0 & 20,3 \\
\hline & $\mathrm{Cz}$ & 6,6 & 9,2 & 5,9 \\
\hline & CF & 58,6 & 69,8 & 73,8 \\
\hline & $S$ & 0,96 & 0,71 & 0,73 \\
\hline \multirow{5}{*}{$\begin{array}{l}\text { Petrográfica } \\
\text { (\% vol.) }\end{array}$} & $\operatorname{Rr}(\%)$ & 0,81 & 1,35 & 1,41 \\
\hline & V & 63,6 & 73,8 & 68,2 \\
\hline & $\mathrm{L}$ & 10,8 & & \\
\hline & I & 18,8 & 16,8 & 25,4 \\
\hline & MM & 6,8 & 9,4 & 6,4 \\
\hline \multicolumn{2}{|l|}{ Fluidez (ddpm) } & 15392 & 944 & 56 \\
\hline \multirow{11}{*}{$\begin{array}{l}\text { Química das Cinzas } \\
\text { (\% em massa) }\end{array}$} & $\mathrm{SiO}_{2}$ & 53,87 & 50,07 & 33,67 \\
\hline & $\mathrm{Al}_{2} \mathrm{O}_{3}$ & 28,96 & 31,27 & 20,43 \\
\hline & $\mathrm{Fe}_{2} \mathrm{O}_{3}$ & 7,48 & 7,29 & 14,02 \\
\hline & $\mathrm{CaO}$ & 1,54 & 2,47 & 15,26 \\
\hline & $\mathrm{K}_{2} \mathrm{O}$ & 2,03 & 2,71 & 1,62 \\
\hline & $\mathrm{Na}_{2} \mathrm{O}$ & 0,57 & 0,57 & 0,46 \\
\hline & $\mathrm{TiO}_{2}$ & 1,59 & 1,70 & 1,04 \\
\hline & $\mathrm{MgO}$ & 0,87 & 0,94 & 1,64 \\
\hline & $\mathrm{MnO}$ & 0,03 & & \\
\hline & $\mathrm{P}_{2} \mathrm{O}_{5}$ & 0,06 & 1,04 & 0,22 \\
\hline & $\mathrm{IB}$ & 0,15 & 0,17 & 0,61 \\
\hline
\end{tabular}

Notação: $M V=$ matéria Volátil, $C z=$ cinzas, $C F=$ carbono fixo, $S=$ enxofre, bs=base seca, $R r=$ reflectância randômica da vitrinita, $\mathrm{V}=$ vitrinita, $\mathrm{L}=$ Liptinita, $\mathrm{I}=$ inertinita e $\mathrm{MM}=$ matéria mineral, $\mathrm{IB}=$ =índice de basicidade.

Para a coqueificação foram utilizadas composições provenientes de carvões individuais e de misturas, sendo elas quatro binárias e três ternárias (Tabela 2).

Tabela 2 - Composições das misturas para fabricação dos coques.

\begin{tabular}{|c|c|c|c|c|c|c|c|c|c|}
\hline \multirow{2}{*}{ CARVÕES } & \multicolumn{7}{|c|}{ Composição das Misturas (\% em massa) } \\
\cline { 2 - 9 } & A & B & C & AB & AC & BC & Abc & aBc & abC \\
\hline A & 100 & & & 50 & 50 & & 50 & 25 & 25 \\
\cline { 1 - 1 } & & \multirow{2}{*}{100} & & 50 & & 50 & 25 & 50 & 25 \\
\cline { 1 - 1 } & & & 100 & & 50 & 50 & 25 & 25 & 50 \\
\hline
\end{tabular}

\subsection{Ensaios de Coqueificação}

Os ensaios foram realizados em um forno elétrico baseado na retorta metálica apresentada na Figura 1. Para o carregamento, as misturas foram quarteadas em 
partes homogêneas, colocadas uma subsequente a outra na retorta e submetidas à compactação até chegar a densidade de carga almejada de $800 \mathrm{~kg} / \mathrm{m}^{3}$. O teste de coqueificação se inicia com o carregamento da retorta no forno pré-aquecido $\left(500^{\circ} \mathrm{C}\right)$, causando uma queda na temperatura. Depois de restabelecida a temperatura de $500^{\circ} \mathrm{C}$, inicia-se um aquecimento com a uma taxa de $3^{\circ} \mathrm{C} / \mathrm{min}$ até 0 centro atingir $1000^{\circ} \mathrm{C}$. Essa temperatura de $1000^{\circ} \mathrm{C}$ é mantida por mais $1 \mathrm{~h}$. Após o término do tempo de ensaio, a retorta foi retirada do forno e o coque imediatamente resfriado em água. Após o apagamento, o coque foi colocado em estufa a $105^{\circ} \mathrm{C}$ por $24 \mathrm{~h}$ sendo posteriormente submetido à caracterização.

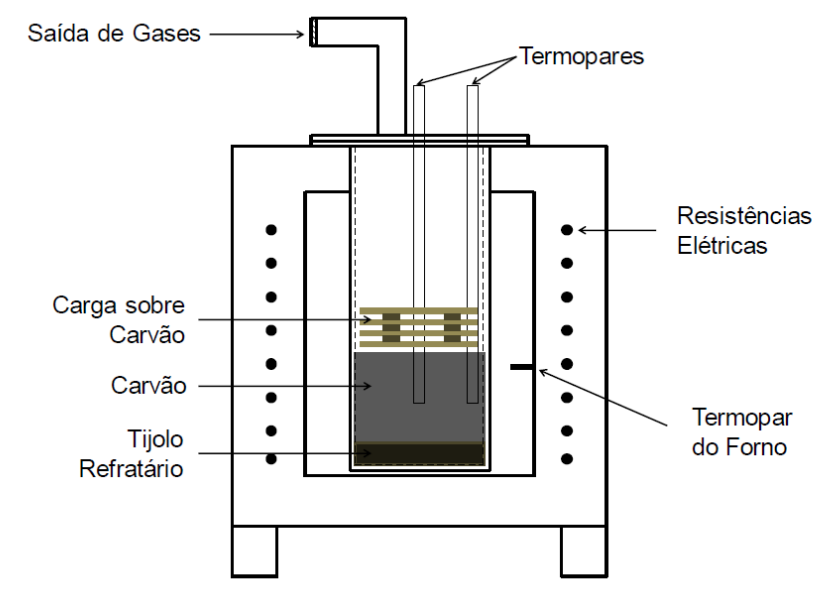

Figura 1 - Esquema de forno e retorta utilizados nos ensaios de coqueificação.

\subsection{Avaliação da Reatividade}

As análises de reatividade ao $\mathrm{CO}_{2}$ foram feitas em termobalança da marca NETZSCH, modelo STA 409 PC Luxx. Para isso utilizou-se uma amostra de 50mg com granulometria entre 1 e $0,2 \mathrm{~mm}$ e um cadinho de alumina tipo copo. O teste consiste em um aquecimento em atmosfera inerte com vazão de $150 \mathrm{~mL} / \mathrm{min}$ de $\mathrm{N}_{2}$ até a temperatura de $1100^{\circ} \mathrm{C}$. Essa temperatura é mantida por 10 minutos com o objetivo de obter a total desvolatilização do coque. Logo após a temperatura é resfriada até $900^{\circ} \mathrm{C}$ e então o gás $\mathrm{N}_{2}$ é substituído por $\mathrm{CO}_{2} \mathrm{com}$ vazão de $200 \mathrm{~mL} / \mathrm{min}$. Após a troca de gases a temperatura é elevada a patamares de 900 , $950,1000,1050$ e $1100^{\circ} \mathrm{C}$. Esse procedimento foi realizado para obter-se a reatividade do coque em diferentes temperaturas, por meio de um único ensaio.

Desta forma foi possível calcular a taxa aparente da reação do coque através da Equação 1:

$$
r_{x}=-\frac{1}{m 0} \cdot \frac{d m}{d t}
$$

Onde $r_{x}\left(m g \cdot \mathrm{mg}^{-1} \mathrm{~s}^{-1}\right)$ é a taxa aparente de reação para as diferentes isotermas testadas, m0 é a massa de coque desvolatilizado e isento de cinzas e dm/dt é a taxa de perda de massa. Para facilitar a visualização dos resultados, a taxa média obtida em cada um dos patamares de temperatura foi multiplicada por um fator de $10^{6}$. Diferentes estudos [7;8] mostram que a taxa aparente de reação obtida em termobalança pode ser comparada a reatividade de coque medida pelo ensaio padronizado CRI-ASTM. Assim, $r_{x}$ foi utilizado como indicador comparativo da reatividade dos coques. 


\subsection{Avaliação da Resistência Mecânica}

Foram feitos dois testes para avaliar a resistência mecânica a frio dos coques produzido sem laboratório. O primeiro trata-se de ensaio em tambor tipo I (Figura 2a), com a massa de amostra de $150 \mathrm{~g}$ e granulometria entre 19 e $21 \mathrm{~mm}$, velocidade de $20 \mathrm{rpm}$ e 600 revoluções. A resistência em tambor tipo I é representada pelo índice 110 , obtido como percentual de amostra retido(malha de 10 $\mathrm{mm}$ ) em relação a massa inicial.

O segundo ensaio de resistência avalia a microresistência do coque. Para isso o equipamento desenvolvido por Blayden e Riley [9] (Figura 2b) foi utilizado. Utilizouse amostra de $20 \mathrm{~g}$ com granulometria entre 3,3 e $6,3 \mathrm{~mm}$.O teste é realizado inserindo-se no interior dos cilindros, além da amostra de coque, 5 esferas metálicas com diâmetro de $15 \mathrm{~mm}$, as quais tem função de aumentar a solicitação mecânica das partículas de coque. A velocidade de rotação foi mantida em $35 \mathrm{rpm}$ até completar 1000 revoluções. A microresistência é representada pelo índice M1, obtido como percentual de amostra retido na malha de $1 \mathrm{~mm}$.
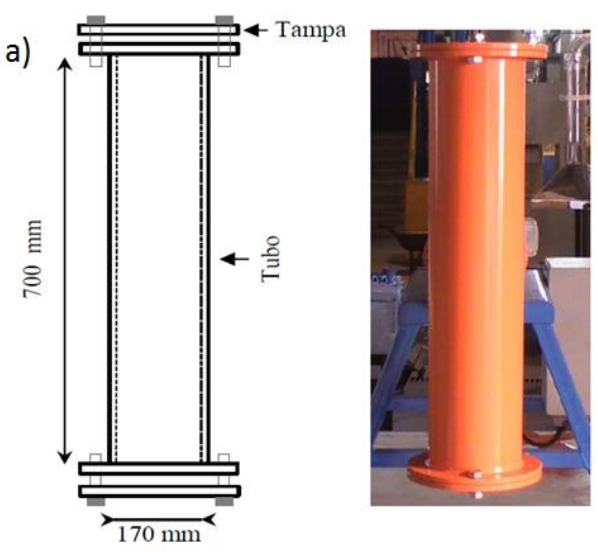

b)
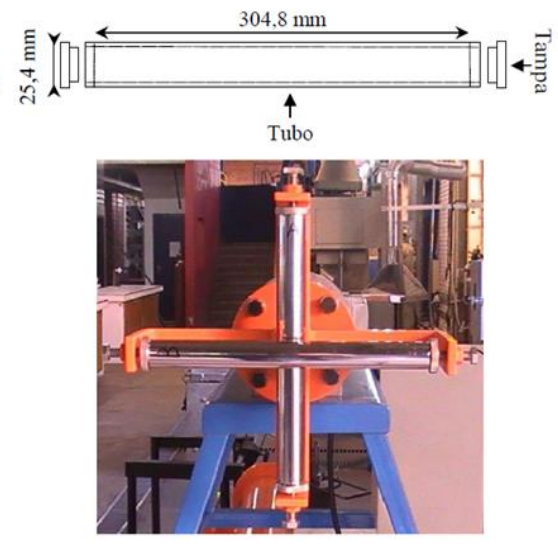

Figura 2 - Aparato para ensaio de resistência mecânica, a) Esquema de tambor tipo I; b)Tubos utilizados para o ensaio de microresistência.

\section{RESULTADOS E DISCUSSÃO}

\subsection{Avaliação da Reatividade dos Coques Produzidos}

A Figura 3 apresenta a variação das taxas de reação em função da temperatura para os coques individuais (Figura 3a) e para os coques produzidos a partir de misturas binárias (Figura $3 b$ ) e ternárias (Figura 3c). Entre os coques individuais nota-se que a amostra obtida a partir do carvão de mais alto rank possui reatividade mais elevada para todas as temperaturas testadas. $\mathrm{Na}$ temperatura de $1100^{\circ} \mathrm{C} \mathrm{O}$ valor da taxa de reação desse coque é 4 vezes mais alta que o coque produzido com carvão de médio rank, que foi o coque de menor reatividade entre todos os coques de estudo. A elevada reatividade desse coque individual está provavelmente associada a maior presença de elementos catalíticos, tais como ferro e cálcio, na composição da sua matéria mineral (Tabela 1). A presença de tais elementos pode resultar na catálise da reação de gaseificação do carbono do coque, elevando assim sua taxa de reação [10]. 

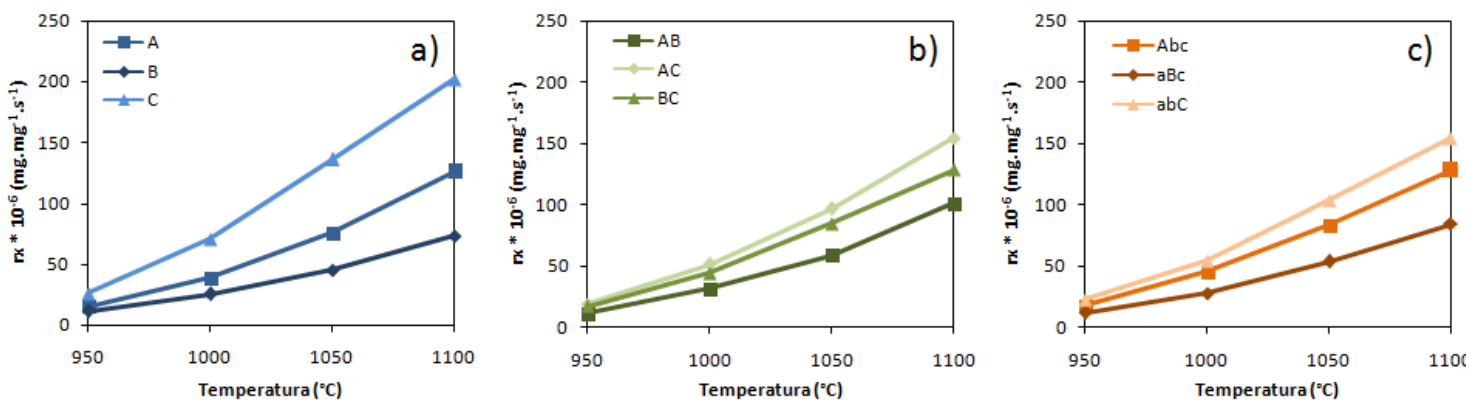

Figura 3 - Variação da reatividade pela temperatura dos coques individuais (a), binários (b) e ternários (c).

Para os coques produzidos através de misturas binárias é possível perceber que 0 coque $A B$ apresenta menor reatividade para todas as temperaturas de estudo. Também percebe-se que para a temperatura de $1100^{\circ} \mathrm{C}$ é possível perceber que a reatividade do coque $A B$ encontra-se em um patamar intermediário de reatividade, ao dos coques individuais provenientes de carvões que compõem a sua mistura. Isto ocorre também para os outros coques.

Para os coques provenientes de misturas ternárias, percebe-se que o coque aBc (25\%A;50\%B;25\%C) apresenta menor reatividade, sendo que, entre as misturas para produção dos coques em estudo, a mistura aBc leva a maior porcentagem de carvão $\mathrm{B}$, que produziu o coque que apresentou menor reatividade. Para os coques de misturas ternárias também é possível perceber que na temperatura de $1100^{\circ} \mathrm{C}$, os valores obtidos se encontram em patamares intermediários aos dos coques individuais produzidos através de carvões contidos em sua mistura.

De modo geral, observa-se que o aumento da temperatura tem influência importante sobre a taxa de reação dos coques. Na temperatura de $950^{\circ} \mathrm{C}$ a taxa de reação de todas as amostras estudadas é praticamente idêntica, com valores na ordem de $1.10^{-5} \mathrm{~s}^{-1}$. As diferenças em termos de reatividade aumentam ao passo que a temperatura também aumenta. Logo o comparativo de reatividade em temperaturas mais altas diferencia as amostra de forma mais importante.

\subsection{Avaliação da Resistência Mecânica dos Coques}

A Figura 4 mostra os resultados obtidos para resistência mecânica a frio dos coques produzidos em laboratório.

Sendo a Figura 4a os valores para os testes de resistência mecânica em tambor tipo I, onde dentre os resultados obtidos, o menor é do coque individual A, produzido pelo carvão com maior matéria volátil. A baixa resistência mecânica deste coque está provavelmente associada formação de um coque esponjoso (observado visualmente). Isso ocorre muito provavelmente devido a combinação de seu alto teor de matéria volátil e fluidez (Tabela 1), resultando em uma porosidade excessiva. Também é possível notar que os valores mais altos pertencem aos coques produzidos através de misturas ternárias. Onde o maior valor obtido foi do coque Abc $(50 \% A ; 25 \% B ; 25 \%$ C), com um I10>82\%.

Pode-se ver na Figura $4 \mathrm{~b}$ que dentre os valores obtidos para os testes de microresistência, o menor pertence também ao coque individual A. Entretanto, acredita-se que nesse caso a excessiva porosidade dessa amostra não possa explicar completamente o resultado, uma vez que o teste de microresitência está altamente associado ao mecanismo de degradação por abrasão. Nota-se também 
que os coques de misturas binárias e ternárias apresentaram valores semelhantes e maiores que os individuais, diferindo-se entre si em 3\% no valor final.
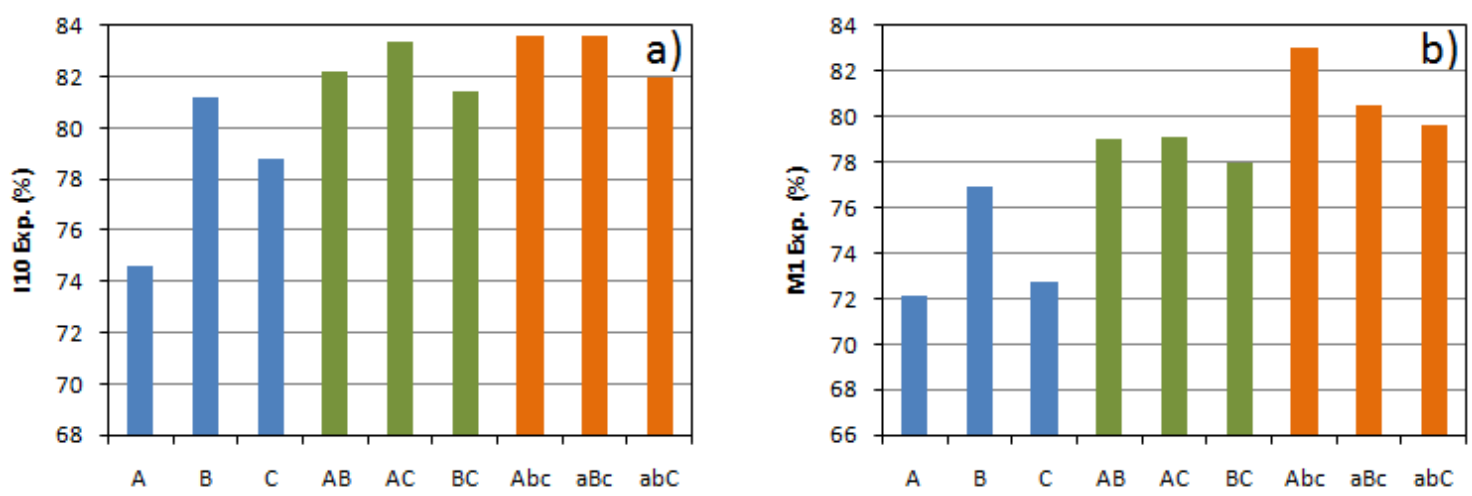

Figura 4 - Índice de resistência mecânica dos coques produzidos em escala laboratorial. A) resistência mecânica em tambor tipo I; b) microresistência.

\subsection{Avaliação dos Modelos Aditivo e de Interações na Previsão de Qualidade do Coque}

O pressuposto da aditividade para uma determinada propriedade no coque parte do princípio que ao se misturar carvões eles não devem interagir. Assim, o indicador de qualidade a ser estimado pode ser calculado a partir das propriedades e frações mássicas dos coques individuais em uma mistura de $n$ componentes. As frações mássicas dos coques são calculadas com base no percentual de cada carvão nas misturas (Tabela 2) e no rendimento de cada carvão. Nesse estudo o rendimento de cada carvão foi obtido pela equação apresentada por Chiu, Y. F. (1983) [11] (rend = 98,89 - $(0,86$ * \% bs Matéria volátil). Sendo assim, é possível calcular as propriedades aditivas através da Equação 2.

$$
V_{\text {aditivo }}=\sum_{i=1}^{n-1} \sum_{j=i+k}^{n} \sum_{k=1}^{n-1} \frac{x_{i} * V_{i}}{x_{i}+x_{j}}
$$

onde $V_{\text {aditivo }}$ é o indicador de qualidade (nesse estudo rx, 110 e M1) do coque produzido a partir de uma mistura de $n$ carvões, $x_{i}$ e $x_{j}$ é a fração mássica de cada coque na mistura e $V_{i}$ é o indicador de qualidade oriundo dos coques individuais.

No entanto, é plausível acreditar que carvões interajam durante o processo de coqueificação, devendo assim modificar as propriedades do coque. $O$ fator " $d_{m}$ binária", proposto por Sakurovs [6] para medir a interação de 2 carvões em relação a suas propriedades plásticas, foi calculado de acordo com a Equação 3 para avaliar a interação dos carvões em relação às propriedades de qualidade dos coques produzidos.

$$
d_{m \text { binária }}=\frac{V_{\text {exp }}-V_{\text {aditivo }}}{x_{i} * x_{j}}
$$

onde $V_{\text {exp }}$ é o valor experimental de uma determinada propriedade do coque

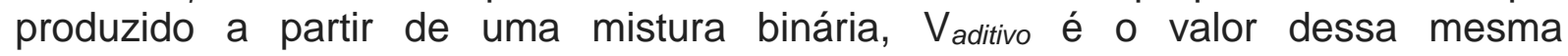
propriedades obtido pela Equação 2 e $x_{i}$ e $x_{j}$ são as frações mássicas dos coques na mistura. Conforme esse mesmo autor [6], ao se determinar experimentalmente o 
valor das interações binárias entre carvões de uma mistura, é possível se estabelecer o valor $d$ para misturas complexas com $n$ componetes. Isto é, para uma mistura ternária de carvões $\mathrm{A}, \mathrm{B}$ e $\mathrm{C}$, é necessário se ter informação dos coques individuais, bem como dos coques binários ( $\mathrm{A}$ e $\mathrm{B}, \mathrm{B}$ e $\mathrm{C}$, e $\mathrm{A}$ e $\mathrm{C}$ ) e a partir disso determinar os $d_{m}$ binários. Assim, calcula-se o fator de interação dos carvões em uma mistura de $n$ carvões ( $\left.d_{\text {mistura }}\right)$ através da Equação 4:

$$
d_{\text {mistura }}=\sum_{i, j>0}^{n} x_{i} * x_{j} * d_{m \text { binário }}
$$

Tendo os valores de $V_{m}$ e dmistura é possível prever a qualidade do coque $\left(V_{k}\right)$, a partir de uma mistura de n carvões, fazendo a soma destes dois valores (Equação 5).

$$
V_{\text {interação }}=V_{\text {aditivo }}+d_{\text {mistura }}
$$

\subsection{Avaliação da Reatividade do Coque pelo Modelo Aditivo e de Interações}

A Figura 5 mostra a correlação entre os valores calculados e experimentais para a taxa de reação dos coques produzidos a partir de misturas binárias e ternárias na temperatura de $1100^{\circ} \mathrm{C}$. A linha pontilhada mostra a relação de idealidade. Observase que os valores calculados são próximos aos valores experimentais, mostrando a aditividade da reatividade dos coques. O maior desvio entre valores calculados e experimentais ocorreu para a mistura $\mathrm{aBc}$, onde o modelo superestima o valor da reatividade.

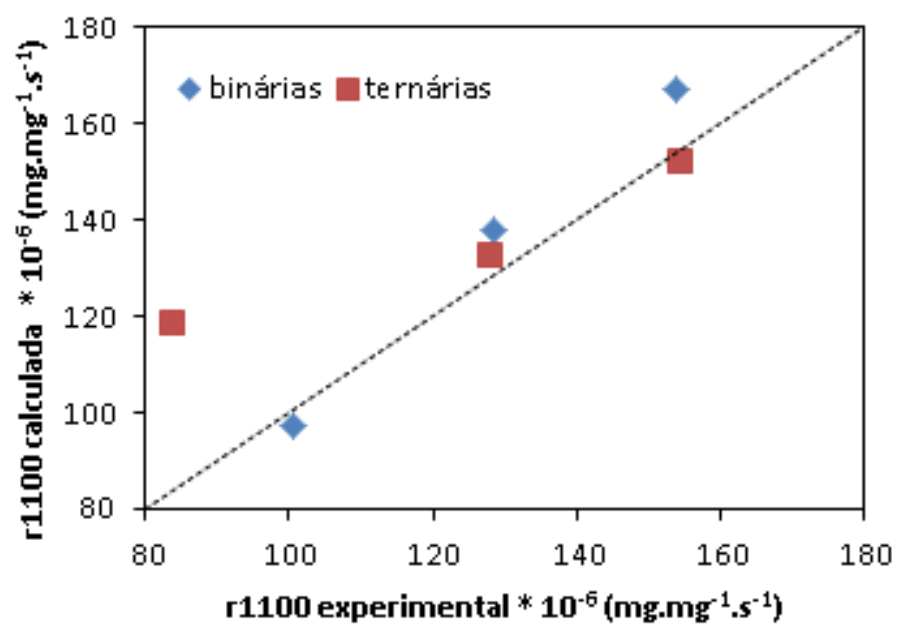

Figura 5 - Correlação entre os valores calculados de acordo com o modelo aditivo e os valores experimentais da taxa de reação dos coques produzidos a partir de misturas binárias e ternárias na temperatura de $1100^{\circ} \mathrm{C}$.

A Figura 6 mostra a correlação dos valores calculados de acordo com o modelo de interação e os valores experimentais para os coques produzidos a partir de misturas ternárias. Observa-se que o modelo representa bem os valores experimentais, mas não oferece ganhos importantes em relação ao modelo aditivo. 


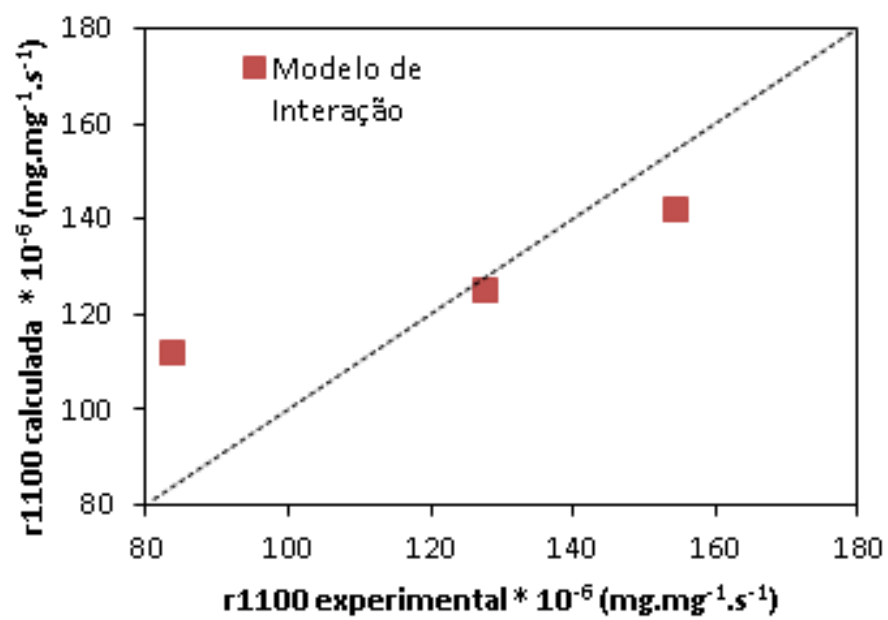

Figura 6 - Correlação entre os valores calculados de acordo com o modelo de interação e os valores experimentais da taxa de reação dos coques produzidos a partir de misturas ternárias na temperatura de $1100^{\circ} \mathrm{C}$.

\subsection{Avaliação da Resistência Mecânica do Coque pelo Modelo Aditivo e de Interações}

A Figura 7 a e b mostra a correlação entre a resistência mecânica dos coques (binários e ternários) calculada de acordo com o modelo aditivo e os valores experimentais dos indicadores 110 e M1, respectivamente. De forma geral, observase que o modelo aditivo não é efetivo para a previsão dos índices de resistência I10 e M1, onde os valores estimados estão todos subestimados em relação aos valores experimentais. Essa falta de correlação indica que a interação entre os carvões foi fundamental para o desenvolvimento da resistência mecânica do coque.
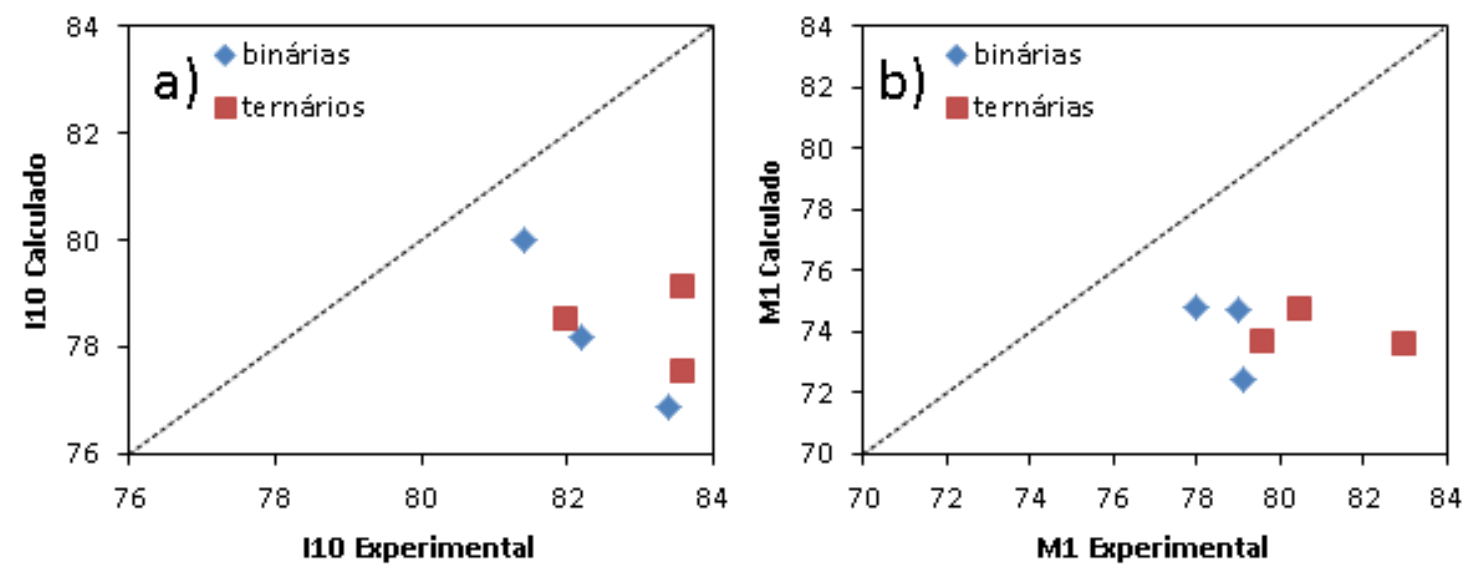

Figura 7 - Correlação entre os valores calculados de acordo com o modelo aditivo e os valores experimentais da resistência mecânica dos coques produzidos a partir de misturas ternárias. a) I10 e b) $\mathrm{M} 1$.

A Figura 8 a e b mostra a correlação entre a resistência mecânica dos coques (ternários) calculada de acordo com o modelo de interação e os valores experimentais dos indicadores $\mathrm{I} 10$ e M1, respectivamente. Tanto para o teste em tambor tipo I (Figura 8a) quanto para microresistência (Figura 8b), percebe-se que os valores calculados pelo modelo de interação apresentam desvios em relação aos dados experimentais. Entretanto, ao comparar-se a eficácia do modelo aditivo e de 
interações é possível perceber uma melhora importante na previsibilidade da resistência com o uso do último modelo.
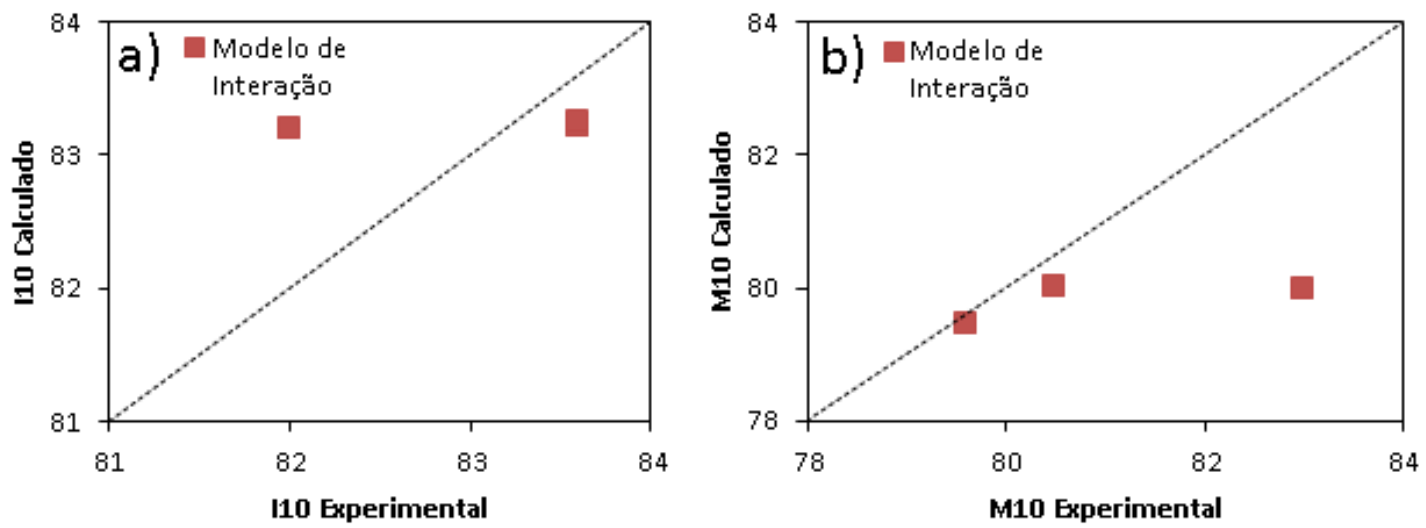

Figura 8 - correlação entre a resistência mecânica dos coques calculada e a experimental levando em conta a interação $d_{\text {mistura }}$ para os indicadores a) 110 e b) M1.

\section{CONCLUSÃo}

A partir da metodologia desenvolvida em laboratório foi possível produzir, avaliar e comparar coques produzidos a partir de diferentes carvões e misturas.

A reatividade dos coques, analisada via ensaios termogravimétricos, mostrou a forte dependência da taxa de reação dos coques com a temperatura de ensaio. $\mathrm{Na}$ temperatura mais alta avaliada $\left(1100^{\circ} \mathrm{C}\right)$, os coques individuais mostrou a seguinte ordem crescente de reatividade: $C>A>B$, coques binários: $A C>B C>A B$ e coques ternários: $a b C>a B c>A b c$.

Em relação à resistência mecânica dos coques avaliada pelo índice 110 os coques individuais mostraram a seguinte ordem crescente: $B>C>A$, coques binários: $A C>A B>B C$ e coques ternários: $A b c=a B c>a b C$. Para 0 índice $M 1$ os coques individuais mostrou a seguinte ordem crescente: $B>C>A$, coques binários: $A C>A B>B C$ e coques ternários: $A b c>a B c>a b C$.

O modelo aditivo para previsão da reatividade em termobalança de coques produzidos a partir de misturas de carvão mostrou boa correlação com os dados experimentais, mostrando o caráter aditivo da propriedade reatividade. $O$ resultado do uso do modelo de interação não apresentou diferenças importantes em relação ao modelo aditivo na previsão da reatividade do coque. Já para resistência mecânica do coque, os resultados calculados a partir do modelo aditivo não foram satisfatórios, com valores fortemente subestimados em relação aos valores experimentais. Em contrapartida, o uso do modelo de interação mostrou valores próximos aos experimentais. Assim, acredita-se que o uso do modelo de interação possa ser utilizado como uma ferramenta interessante na seleção e mistura de carvões.

\section{Agradecimentos}

O autor agradece ao CNPq pela bolsa de iniciação e a Gerdau pelo fornecimento dos carvões. 


\section{REFERÊNCIAS}

1 NAKAMURA, N.; TOGINO, Y.; TATEOKA, M. Behaviour of coke in large blast furnace.Coal, Coke and Blast Furnace.The Metals Society, London, p.1-18, 1977.

2 DÍEZ M.A.; ALVAREZ R.; BARRIOCANAL C. Coal for metallurgical coke production: predictions of coke quality and future requirements for cokemaking. International Journal of Coal Geology, V. 50P. 389-412, 2002.

3 CHIU, Y.F. Study of coke petrography and factors affecting coke reactivity. Ironmaking Steelmaking, , v. 5, p. 196-199, 1982.

4 ÁLVAREZ, R.; DÍEZ, M.A.; BARRIOCANAL, C.; DÍAZ-FAES, E.; CIMADEVILLA, J.L.G. An approach to blast furnace quality prediction. Fuel, 2007

5 Abe Y, Nishi T, Suzuki S, Shiki Ch, Katahira H. Control of measure of post reaction strength of coke with the same cold strength of coke. Trans ISIJ 1983;23:PB-272.

6 SAKUROVS, R. A method for identifying interactions between coals in blends. Fuel Vol. 76, No. 7, pp. 623-624, 1997.

7 F. L. G. E Oliveira, V. Zymla, N. Jannotti, Reactivity of coke used in the electric furnace for ferroalloys production, 3rd International Meeting on Ironmaking (2008) 1154-1164.

8 J. I. Rodero, J. Sancho-Gorostiaga, M. Ordiales, D. Fernandez-Gonzalez, J. Mochon, I. Ruiz-Bustinza, A. Fuentes and L. F. Verdeja, Blast furnace and metallurgical coke's reactivity and its determination by thermal gravimetric analysis, IronmakSteelmak 42 (2015) 618-625.

9 BLAYDEN, H.E.; NOBLE, W.; RILEY, H.L. The influence of carbonizing conditions on coke properties. Part I. Mechanical Pressure. Jour. Iron and Steel Inst. V. 136, p. 47-76, 1937.

10 W.W. Gill, N.A. Brown, C.D.A. Coin, M.R. Mahoney, The influence of ash on the weakening of coke. Ironmaking Conference Proceedings. 44 (1985) 233-238.

11 Y. F. Chiu and M. T. Hong Influence of volatile matter and deposited carbon on coke yield from coals. R \& D Department, China Steel Corporation. 1983. 